Sensors and Actuators B 169 (2012) 188- 194, http://dx.doi.org/10.1016/j.snb.2012.04.064

\title{
DNA-based Biosensor Platforms for the Detection of TP53 Mutation
}

Z. Altintas ${ }^{\mathrm{a}, \mathrm{b}}$ and I.E.Tothill ${ }^{\mathrm{a}^{*}}$

${ }^{a}$ Cranfield University, Cranfield Health, Cranfield, Bedfordshire, MK43 OAL, England, UK.

${ }^{\mathrm{b}}$ Faculty of Natural Sciences and Engineering, Sabancı University, Istanbul, 34956, Turkey

* Corresponding author: I.E. Tothill. Tel: +44 (0) 7500766487, Fax: +44 (0) 1234 758380 E-mail: i.tothill@cranfield.ac.uk 


\section{Abstract}

A DNA-based assay for the detection of one-point mutation in TP53 gene, responsible for lung cancer, was developed using a surface plasmon resonance (SPR) and a quartz crystal microbalance (QCM) biosensor systems. Amine coupling was employed for the immobilization of NeutrAvidin on thiol- derivatised surface to capture the biotinylated target sequence. Two targets sequences and one control DNA sequence were investigated including, a fully complementary (30 mer), onepoint mutation and a non-complimentary DNA using hybridization with a detection probe strand (27 mer). The most appropriate surface coating was also examined for both sensor platforms with hybridization and single nucleotide polymorphism (SNP) detection efficiency were then compare. A $0.03-2 \mu \mathrm{M}$ concentration range of detection probe was detected using the SPR and QCM sensors on wild and mutant type target surfaces. The linear regression analysis was performed for both sensors resulting in a $R^{2}$ value for the SPR assay of 0.985 and 0.993 for perfect and mismatch reaction and of 0.978 and 0.976 for the QCM assay, respectively. The obtained results demonstrate that the used approach represents a very promising future method for the detection of one-point mutation in genetic-based health problem with highly sensitive, specific, and real-time analysis.

Keywords: Surface plasmon resonance, SPR, quartz crystal microbalance, QCM, DNA mutation, TP53, cancer. 


\section{Introduction}

The human genome is subjected to mutations during the lifecycle due to several types of change in the DNA. The mutation may arise from viruses, radiation, transposons, mutagenic chemical or any errors that occur during DNA replication or meiosis process in the cell [1,2]. Many mutations have no effect on phenotype or health whereas some mutations cause crucial diseases such as cancer or cardiac diseases and therefore, better understanding of mutation effects on phenotype is very important part of genetic studies [3,4]. Various methodologies have been currently employed for the detection of mutation in cancer cases and TP53 gene is one of the most investigated genes due to its relation with many types of human cancers and cells pathways [5]. Most regions of this gene have been investigated using PCR-based techniques [6], sequencing with microarray technology [7], and fluorescence-based techniques [8].

As a strong alternative to the above techniques, biosensor technology has recently constituted a real-time, label-free, sensitive and rapid approach to be used for DNA mutation detection. Various types of sensors have been employed for hybridization assay studies including electrochemical, optical and piezoelectric based sensors showing high efficiency [9-11].

A wide range of investigations have been conducted regarding p53 gene mutations in cancer cases; most of these are genetic or medicine-based studies where the detection of mutations have been mainly achieved using common traditional techniques and tools. Biosensor technologies through novel approaches provides a strong alternative to generally used methodologies for the detection of hereditary diseases; moreover, the technology constitutes a promising future methods of testing in hospitals due to the real-time, rapid and highly specific nature of biosensors [12-14]. However, to obtain a sensitive and highly specific detection method for cancer markers, development of sensor platforms and surfaces are the crucial points in this research field. Both protein and gene markers for many diseases have been studied with biosensor technologies [15-19]. Though there have been very limited published research on SNP detection, the published data for cancer diagnosis has recently shown a rapid increase [20-23]. Most published research in this area 
use commercially available nucleotide products and show a promising approach for the detection of gene-related diseases in human patient samples.

In the present study, SPR based sensor chip (Biacore 3000) and a new QCMbased sensors (QCMA-1, Sierra Sensors) were employed to develop a hybridization and SNP detection assay for the detection of TP53 mutations. Due to the importance of the surface chemistry used on sensor surface and its role in increasing the capacity of biomarker detection, a surface selection procedure was initially performed on the SPR bare gold sensor chip and the surface with the best output for both single-strand DNA probe capture and DNA hybridization assays was then selected for use for both the SPR and QCM assay development [24]. A 1\% MUDA (1\% $2 \mathrm{mM}$ MUDA and 99\% $2 \mathrm{mM}$ MUDO) coated sensor surface with an immobilized NeutrAvidin using EDC/NHS chemistry was therefore used in this study. After obtaining prospective success from the SPR biosensor for SNP detection, the same procedure was then transferred to a QCM-based sensor platform. However, the $1 \%$ MUDA coated QCM sensor surface did not give good results and hence three other different ethanolic solution of MUDA were examined and the highest response was achieved by coating of the surface with 100\% MUDA (2 mM MUDA, MUDO free). Through two separate sensor platforms, SNP detection in codon 248 of p53 gene for lung cancer diagnosis was researched using commercially available oligonucleotides. The mutation in codon 248 and 273 shows the commonest frequency for all lung cancer cases. An efficient DNA capture and hybridization were initially obtained and then SNP detection was examined via both sensor types.

\section{Experimental}

\subsection{Reagents}

Mercaptoundecanoic acid (MUDA), mercaptoundecanol (MUDO), spectrophotometric grade ethanol, phosphate buffered saline (PBS, $0.01 \mathrm{M}$ phosphate buffer, $0.0027 \mathrm{M}$ potassium chloride and $0.137 \mathrm{M}$ sodium chloride, $\mathrm{pH}$ 7.4), and all oligonucleotides were purchased from Sigma-Aldrich (Poole, UK). 1ethyl-3-(3-dimethylaminopropyl)-carbodiimide (EDC) and NeutrAvidin (NA) and EZlink-sulfo-NHS-Biotin were purchased from Pierce-Thermo Scientific (Cramblington, 
UK). Oxygen free nitrogen was purchased from BOC (Manchester, UK). Ultrapure water (18 M $\Omega \mathrm{cm}-1$ ) was obtained from a Milli-Q water system (Millipore Corp., Tokyo, Japan). Tris- $\mathrm{HCl}, \mathrm{NaCl}$, EDTA and Tween 20 were purchased from SigmaAldrich (Poole, UK).

Oligonucleotides were bought from Sigma-Genosis (Poole, UK). The used oligonucleotides sequences; Wild-type target: 5' [Biotin] CAGCTTTGAGGTGAGTGTTTGTGCCTGTCC, mutant-type target: 5' [Biotin] CAGCTTTGAGGTGATTGTTTGTGCCTGTCC, scrambled DNA sequence: 5' [Biotin] CCATCGGCATGTACCGTATCGGCGCGT, detection probe: 5' [Biotin] GGACAGGCACAAACA ATCACCTCAAAG. Here the detection probe was selected with biotin for possible inclusion of an amplification step, however, a probe without biotin should otherwise be used in the assay.

\subsection{Instrumentations}

A Biacore 3000 instrument with bare gold sensor chips (Biacore, GE Healthcare, Uppsala, Sweden) and a fully automated QCMA-1 system with bare gold sensor chips (Sierra Sensors GmbH, Hamburg, Germany) were used in this study. Biacore sensor has four sensing spots whilst QCMA-1 has two sensing spots. The operating temperature of the assays was $20^{\circ} \mathrm{C}$ and the flow rate of the buffer was $10 \mu \mathrm{min}^{-1}$ for the Biacore 3000 whereas for the QCMA-1 a $25^{\circ} \mathrm{C}$ and $80 \mu$ min $^{-1}$ were used throughout the assay. Three sensing spot of the Biacore 3000 sensor platform were used; each of wild-type, mutant-type and scrambled DNA sequences were injected to the separate sensing spot to perform simultaneous assay on both target and control surfaces. On the other hand, since QCMA-1 has only 2 sensing spot, one of the spots was always used as control surface through injecting scrambled DNA surface on the spot whereas another spot was employed for either wild-type target or mutanttype target in separate experiments. 


\subsection{Procedures}

\subsubsection{Sensor surface preparation}

The SPR sensor chip surfaces of the Biacore 3000 instrument were prepared by immobilising NeutrAvidin on separate three sensing spots. The immobilisation of NeutrAvidin was obtained using conventional amine coupling chemistry, simply named as EDC/NHS chemistry and previously degassed PBS was used as running buffer during the immobilisation process of NeutrAvidin. First, the sensor chips were coated with the mixture of MUDA and MUDO to obtain a self assembled monolayer (SAM) on the bare gold sensor surface. Both thiol solutions were prepared in pure ethanol as $2 \mathrm{mM}$ stocks and 1\% MUDA (1\% $2 \mathrm{mM}$ MUDA and 99\% $2 \mathrm{mM} \mathrm{MUDO)}$ was then prepared using these stock solutions. To immobilize NeutrAvidin, the SAM coated sensor chip was docked to the Biacore instrument and then the instrument was primed with PBS running buffer. After priming, the sensor chip surface was initially cleaned by a mixed solution of $1 \%$ Triton and $100 \mathrm{mM} \mathrm{NaOH}$ through injection of this solution to the sensing spots for 1 minute to prevent from any particles on the surface prior to activation of MUDA coated surface with EDC/NHS. Sensor surfaces were activated with a $1: 1$ mixture of $400 \mathrm{mM}$ EDC and $100 \mathrm{mM}$ NHS that previously prepared and stocked at $-20^{\circ} \mathrm{C}$, and mixed immediately before the experiments. The mixed solution of EDC/NHS was injected to the three sensor surfaces for $3 \mathrm{~min}$. A $100 \mathrm{mg} \mathrm{ml}^{-1}$ NeutrAvidin (diluted in 1 X PBS buffer) was then injected to the surfaces for two times as 5 and 3 minutes to immobilize NeutrAvidin on surfaces. At the end of immobilization, $1 \mathrm{M}$ ethanolamine $(\mathrm{pH}: 8.5)$ was injected to the sensor chip surface during 3 minutes for blocking non-reacted NHS esters to prevent from non-specific bindings.

The QCM bare gold chips were firstly coated with 100\% MUDA solution (2 mM MUDA; MUDO free) during overnight incubation under dark condition at room temperature after cleaning of the QCMA- 1 chips by nitrogen plasma. The coated chips were then docked to the QCMA-1 instrument and $\mathrm{dH}_{2} \mathrm{O}$ was changed to PBS buffer for priming and then immobilization of NeutrAvidin. The surfaces of the chips were firstly washed with a mix solution of Triton and $\mathrm{NaOH}$ by injection of the solution for 1 minute. EDC/NHS was then injected to the two separate sensing spots of the instrument to activate the surface. A 5 minutes injection of $100 \mu \mathrm{g} \mathrm{ml}^{-1}$ NeutrAvidin 
was then performed and $1 \mathrm{M}$ ethanolamine was then used to cap non-reacted NHS esters on the surface during 3 minutes to complete the immobilisation step of the assay. Degassed PBS was used as running buffer during the immobilisation period.

\subsubsection{DNA capture and hybridization assays for SNP detection}

Three different surface sequences (wild-type target, mutant-type target and scrambled DNA Sequence) for DNA capture and one detection probe for hybridization were used. Wild-type target included the region of p53 gene which is responsible for codon 248 and mutant-type target had the same DNA sequence of the wild-type target except for a particular base that converse from $G$ to $T$ when a SNP occurs due to lung cancer. Scrambled surface had a completely different oligonucleotide sequence from other target sequences and it was used to obtain a control surface for hybridization reaction, since the detection probe was not complementary for this surface probe.

Moreover, detection probe was designed according to the sequence of mutanttype target that means it shows a perfect match with mutated sequence whereas it has a mismatch on wild-type target due to one nucleotide change. Therefore, the expected results from the hybridization reaction should be higher on the mutant-type target than the wild type and the obtained hybridization on the scrambled sequence would be at approximately zero level. The principle of SNP detection according to the used design in this study is represented in Fig.1. After NeutrAvidin immobilization, running buffer was changed from PBS to Tris-Tween (10 mM Tris- $\mathrm{HCl}, 1 \mathrm{mM}$ EDTA, $150 \mathrm{mM} \mathrm{NaCl}, 2.5 \mathrm{ml}$ 0.05\% Tween 20, pH: 7.0) for DNA capture and hybridization assay. Tris-Tween buffer was degassed and used as a running and dilution buffer. With changing PBS to Tris-Tween running buffer, the Biacore 3000 instrument was primed and then single-stranded surface sequences (wild-type target, mutant-type target and scrambled control) were injected to the separate sensing spots of the sensor using a $50 \mu \mathrm{M}$ concentration in all experiments and each was injected to the particular sensing spots for 5 minutes and the $\mathrm{RU}$ changes were recorded during this period. After injection of all capture sequences and the immobilisation was completed, biotin blocking solution $(\mathrm{pH}: 7.6,11.5 \mathrm{mg}$ was diluted in $50 \mathrm{ml}$ as a stock solution) and was injected to the sensor spots for 1 minute to cap sequence-free 
NeutrAvidin molecules on the sensor surface and to prevent non-specific binding during the hybridization stage of the assay. This proved to be highly efficient in capping all unoccupied NeutrAvidin molecules. The detection probe was then injected and the hybridization reaction took place. The concentration series used for the detection probe was in the range of $0.03-2 \mu \mathrm{M}$ in the Biacore 3000 assays. Both surface sequences and detection probe were diluted in Tris-Tween buffer and this was also used as the running buffer during the capture of surface sequences on NeutrAvidin immobilized sensor surface until the completion of the hybridization reaction using detection probe. During the serial hybridization reactions, the surface was regenerated with $100 \mathrm{mM} \mathrm{HCl}$ without loss of activity. Each concentration of detection probe was injected to three sensing spots simultaneously for 5 minutes and the $\mathrm{RU}$ change was recorded during this time. Here, three sensing spots of the SPR sensor were only used (one for the wild type target surface, the second for the mutant target surface and the third was employed to immobilised the scrambled sequence that provided control surface throughout the assays).

A similar method was followed for the DNA capture and hybridization assays using the QCMA-1 instrument. However, since QCMA-1 has only two sensing spots, one was always used for scrambled sequence capture on the surface to obtain a control surface whereas the other spot was used for injection of either mutant-type or wild-type targets. To stabilize the experimental conditions to the utmost, target surfaces (either wild-type or mutant-type) were injected to first sensing spot of the instrument whilst scrambled sequence was injected to the second spot. The injection time of each capture sequence was 5 minutes and after the capture of sequences, non-capped NeutrAvidin molecules were blocked by 1 minute injection of biotin solution (prepared as previously). After the target/control sequence capture of the assay completed, hybridization was started with detection probe injection. The same concentration range of detection probe was used here as that applied on the SPR sensor with the Biacore 3000 instrument and the dilution of the concentration series was done using Tris-Tween buffer. After each 5 minutes injection of detection probe depending on concentration used, the surface was regenerated with $100 \mathrm{mM} \mathrm{HCl}$ solution during 1 minute injection. 


\section{Results and discussion}

\subsection{Development of the SPR assay}

The Biacore 3000 instrument was used as the biosensor platform for SNP detection. Bare gold surfaces of the SPR sensor chips were coated with $1 \%$ MUDA to obtain a self assembly monolayer for NeutrAvidin immobilization. The chip was then docked to the system and primed with buffer flow. NeutrAvidin was immobilized to the surface through conventional EDC/NHS chemistry. The recorded RU change for NeutrAvidin immobilization was $3661 \pm 701 \mathrm{RU}(\mathrm{n}=30)$ (Fig. 2a). Due to adequate immobilization level of NeutrAvidin, the assay was followed by target sequence capture. A $50 \mu \mathrm{M}$ concentration of wild-type, mutant-type and scrambled surface sequences were injected to separate sensing spots of the instrument for 5 minutes (Fig. 2b). Mutant-type and wild-type DNA sequences were employed as target surfaces whilst scrambled sequence was used as a control surface to understand the specificity of the interaction. Mutant-type target captured sensors produced $525 \pm 55$ $R U$ change $(n=10)$, whereas the recorded response for the wild-type and scrambled probes were $539 \pm 71,564 \pm 45 \mathrm{RU}(\mathrm{n}=10)$, respectively (Fig. 2c). The produced RU change of the sensor for each capture sequence was similar and this was an expected result since all sequences (both target sequences and scrambled sequence) had approximately the same nucleotide length. The difference between targets and scrambled sequence was slightly higher since the oligonucleotide content was completely different. Moreover, \%GC content and secondary structure of oligonucleotides may affect the binding capacity on the sensor [23]. \%GC content of wild, mutant and scrambled surface sequences were 56.7, 50 and 63, respectively, and the probability of forming secondary structure for targets was weak whereas it was at moderate level for scrambled sequence.

With obtaining an adequate signal for the NeutrAvidin immobilization and DNA capture from the sensor surfaces, the assays were then followed by conducting the hybridization reaction. Detection probe designed according to the mutant-type target surface to achieve perfect match was employed in the concentration range of 0.03-2 $\mu \mathrm{M}$. The results showed gradual increases in the $\mathrm{RU}$ values were achieved when the concentration increased from $0.03 \mu \mathrm{M}$ to $1 \mu \mathrm{M}$. However, the obtained $\mathrm{RU}$ change 
after $1 \mu \mathrm{M}$ concentration of detection probe generally showed a study state response (Fig. 3a and b). In order to show the appropriate concentration to be used for detection without competition, both $1 \mu \mathrm{M}$ and $2 \mu \mathrm{M}$ were used as higher concentrations and the shown data in this study represents the overall results of the experiments for these concentrations. Hybridization reaction was successfully obtained in selected concentration range of detection probe using Biacore 3000 instrument. Furthermore, non-specific binding on the control surface was recorded at zero level whereas a high response was obtained on the target surfaces including the wild-type and the mutant-type capture sequences. A clear difference was achieved between the two target surfaces in which hybridization on mutant-type capture sequence gave higher response as $R U$ change than the wild-type surface due to perfect match with the detection probe, whilst the wild-type target had a mismatch due to single nucleotide polymorphism (SNP).

The detection of each concentration was continued only for 5 minutes including injection of the sample and recording of the $\mathrm{RU}$ change. A complete regeneration of the surface for the next binding was carried out using $0.1 \mathrm{M} \mathrm{HCl}$ solution. A linear increase was observed until a $1 \mu \mathrm{M}$ concentration of complementary detection probe was added. Furthermore, low concentration of hybridization probe $(0.03 \mu \mathrm{M})$ was successfully detected with a clear and specific response produced by the SPR-based biosensor platform. Hagihara and colleagues, studied the detection of G-A mismatches using an SPR sensor and showed that a concentration range of 0.13-1 $\mu \mathrm{M}$ DNA can be detected using interaction of naphthyrine-azaquinolone hybrid molecules on the surface instead of NeutrAvidin-biotin interaction by applying EDC/NHS surface chemistry method [25]. Our results using the Biacore 3000 showed a linear response with $R^{2}$ values of 0.98 and 0.99 for perfect match on mutant-type surface probe and SNP on wild-type surface, respectively (Fig. 3c). The expected output was achieved from this work for SNP detection using the Biacore 3000 instrument and the sensor surface developed in this study. The use of the appropriate surface chemistry and assay method on the sensor chip constitute the most critical step for SNP detection [24]. Though a clear hybridization was obtained from different type of surfaces, the efficiency of the binding must be very high to achieve single-nucleotide polymorphism detection since it requires higher sensitivity than that required for the detection of a piece of DNA or RNA sequence. 


\subsection{Development of the QCM assays}

Here, a QCMA-1 biosensor system was also used to evaluate the hybridization efficiency of oligonucleotides using a QCM-based sensor and to compare results of SNP detection with the Biacore 3000 device. Different \% concentrations of $2 \mathrm{mM}$ MUDA were examined for surface chemistry modification on the QCM sensor surface. A $100 \%$ MUDA (2 mM MUDA, MUDO free) was found to be the optimal concentration to be used for the assay development on the QCM bare gold sensor chip. This enabled a self assembled monolayer to be formed which gave the best output for DNA capture on the surface with each single-stranded surface probe (data not shown). A $100 \mathrm{\mu g} \mathrm{ml}^{-1}$ neutravidin was initially immobilized to two separate sensing spots of the instrument using EDC/NHS chemistry after the sensor chip surface was washed with mixed solution of $1 \%$ Triton and $100 \mathrm{mM} \mathrm{NaOH}$ during a1 minute injection. NeutrAvidin immobilized sensors produced an average frequency change of $773 \pm 95 \mathrm{~Hz}(n=10)$.

In each assay, the first spot was used for either wild or mutant-type targetss and the second spot was always employed for capture of scrambled sequence as a control surface. A $50 \mu \mathrm{M}$ concentration of each surface sequence was injected to the particular sensing spot of the sensor during 5 minutes and DNA sequence-free areas of the surface were then blocked by a 1 minute injection of biotin solution to cap the free sites on the neutravidin surface. The recorded frequency changes were obtained as $233 \pm 28(n=10), 237 \pm 27(n=10)$ and $220 \pm 29 \mathrm{~Hz}(n=10)$ for mutant, wild and scrambled surfaces respectively (Fig. 4a). The produced frequency values of the sensor for the two target surfaces (wild and mutant) were close to each other, with a lower frequency achieved for the scrambled surface since the targets had the same oligonucleotide sequences except for one base which was $G$ in wild-type target instead of $\mathrm{T}$.

The DNA capture assay was followed by hybridization using the detection probe. This probe was designed as fully complementary to mutant-type single stranded target surface; therefore, a higher response was expected from binding of detection probe on the mutant-type target surface than the wild-type on which a mismatch existed due to SNP. The studied concentration range of detection probe was $0.03-2$ 
$\mu \mathrm{M}$ and a clear decrease was observed after $1 \mu \mathrm{M}$ because of competition between the DNA molecules. The specificity of the assays was very high since the detection probe did not bind to the control surface as seen in Fig. 4b. Each concentration of hybridization probe was injected to both sensing spots for 5 minutes and the sensor surfaces were regenerated by a 1 minute injection of $0.1 \mathrm{M} \mathrm{HCl}$.

Furthermore, a gradual increase was observed for the hybridization depending on the concentration on both wild and mutant-type target surfaces with a high specificity. A $0.03 \mu \mathrm{M}$ concentration of hybridization probe could be detected by QCMA-1 sensor. The recorded frequency change for $0.03 \mu \mathrm{M}$ was $26 \pm 10 \mathrm{~Hz}(n=10)$ whereas non-specific binding of the detection probe on the control surface was only $2 \pm 1 \mathrm{~Hz}$ $(n=10)$ (Fig. 4b).

The expected hybridization potential was successfully achieved with a linear concentration range of the detection probe on both wild and mutant target surfaces with high specificity. This achievement demonstrated that the QCM-based biosensor is very suitable for the detection of nucleic acids, particularly for DNA molecules. Moreover, the aim of the study was to detect the one-point mutation of lung cancer for early diagnosis; thus, all collected data for each target surface was evaluated and compared to understand if a difference exists or not between the hybridization of detection probe on wild and mutant-type target sequences. According to our design for the artificial DNA probe, a higher response was expected from the interaction of mutant-type target and detection probes due to the perfect match between the two sequences. The overall results show that one-point mutation was clearly detected through the QCMA-1 instrument, when concentrations of above $0.5 \mu \mathrm{M}$ were used. The correlation efficient of SNP detection on target surfaces in the linear dynamic range of $0.03-2 \mu \mathrm{M}$ is shown in Fig. 4c.

When the reproducibility and effectiveness of the two sensor platforms were compared for SNP detection, the SPR-based biosensor gave better results than the QCM-based sensor, though the results obtained from both platforms showed similarity. Since the capture of targets on the sensor chip surfaces were stronger in the Biacore 3000 than QCMA-1 the obtained responses for hybridization in the 
sensors was parallel to the amount of target DNA capture on the sensor chip surfaces (Fig. 5a and b).

This study has shown a highly promising future for diagnosis of various diseases that has genetic marker by biosensor technology. Although there are a number of published studies in the literature regarding the detection of nucleotides using SPRbased sensors, to date the success for SNP detection have been very limited by this technology. Jiang et al. used SPR-technology to detect a mutation in the region of p53 gene that corresponding to codon 248 [26] and they used previously optimized analytical parameters of two different SPR-based sensors developed by Wang et al [27]. In addition, a published study about SNP genotyping for increased risk of venous thrombo-embolism was performed with an optical sensor in which optical thin-film biosensor chips were used and a ligation-based assay was applied on optical-detection platform instead of fluorescence-detection methods with a more sensitive and rapid approach. However, the method used in our work takes only 5 minutes for the detection of the target sequence whereas the applied procedure used in other work took up to 45 minutes [20].

Other researchers, such as Nakatani et al [28], developed a method to detect SNP using an SPR sensor with a similar procedure when compared with our study. However, their mutation analysis was based on the binding between naphthyridine and G-G mismatch. The method detects a single nucleotide mutation from $\mathrm{G}$ to $\mathrm{C}$ or $\mathrm{C}$ to $\mathrm{G}$, and uses molecular modelling for the discovery of low-molecular-weight ligands of dimeric naphthyridines 1 and 2 which specifically bind with high affinity to the G-G mismatch, irrespective of the sequence flanking the mismatch. Their results suggested that the G-G mismatch would be significantly stabilized when two naphthyridine intercalators simultaneously bind to both $\mathrm{G}$ bases within the base stacks of the DNA duplex. They investigated 27-mer DNA duplexes in the concentration range of $0.125-1 \mu \mathrm{M}$. The work we presented in this paper showed a broader dynamic range of the method we developed for SNP analysis (0.03-2 $\mu \mathrm{M})$, with lower detection limit which is important for the detection of real patient samples. Moreover, there is no need to use specific base-pair mismatch in our study when compared to Nakatani et al work [28]. Okumura et al [29], developed a method for a K-ras point mutation detection using a sandwich assay enhanced with hydrogel nanospheres and surface plasmon resonance imaging technique. In their study, a 
homobifunctional alkane dithiol was first adsorbed on Au film and ethyleneglycol diglycidylether (EGDE) was then reacted to insert the ethyleneglycol moiety, which can suppress nonspecific adsorption during SPR analysis. Streptavidin was then immobilized on EGDE using tosyl chloride activation. Biotinylated DNA ligands were then bound to the streptavidin modified surface to produce the DNA arrays. In the SPR analysis, the DNA analyte was exposed on the DNA array and hybridized with the immobilized DNA probes. Subsequently, the hydrogel nanospheres conjugated with DNA probes were bound to the DNA analytes in a sandwich configuration which led to SPR signal enhancement and enabled the discrimination of a K-ras point mutation in the SPR difference image. This investigation has shown similar procedure with our study in which covalent interaction of the steptavidin-biotin molecules was used to produce DNA arrays. However, the researchers used hydogel nanosheres to enhance the signal which make their procedure more complex and expensive when compared to our method. Dell'Atti et al [30] also investigated TP53 point mutations using QCM-based sensor. In the study, a DNA-based piezoelectric biosensor for the detection of the TP53 gene mutation at codon 248 was reported. A biotinylated probe was immobilised on the sensor surface via dextran-streptavidin modified surfaces. The sensor was optimised using synthetic oligonucleotides and . applied to polymerase chain reaction (PCR)-amplified real samples of DNA extracted from two cell lines, one normal (wild-type) and one mutated, carrying the mutation at codon 248 of the TP53 gene. The results obtained demonstrate that the DNA-based piezoelectric biosensor is able to detect the point mutations in PCR-amplified samples showing the potentialities of this approach for routine analysis [30]. The method used is similar to our present work. However, we used the specific interaction between neutravidin and biotin instead of dextran-streptavidin; and we successfully investigated a broader dynamic range of the samples with an excellent specificity and sensitivity in both SPR and QCM-based systems. 


\section{CONCLUSIONS}

In this study, single nucleotide polymorphism (SNP) detection was investigated through the use of two different sensor platforms (SPR and QCM) based on label free technology using commercially available oligonucleotides. The main aim of the study was to demonstrate the importance of biosensor technologies for diagnosis of molecular biomarkers for cancer through the detection of one-point mutations that occurs mostly in various region of p53 gene. A highly specific and sensitive hybridization reaction was successfully achieved in the concentration range of $0.03-2$ $\mu \mathrm{M}$ detection probe through a rapid, real-time assay approach. Furthermore, onepoint mutation could be significantly detected using interactions between target surface probes and detection probe by the Biacore 3000 and QCMA-1 instruments. When the reproducibility and effectiveness of the two developed assays on the sensors were compared, the SPR-based assay gave better results than the QCMbased assay using the format applied in this study, though the obtained results from both platforms showed high similarity. Since the capture of target sequences on the sensor chip surface was stronger in the Biacore 3000 than the QCMA-1, the obtained hybridization responses gave parallel results. The performed experimental procedure was neither time-consuming nor with labelling; therefore, the achieved results indicate that both sensors can be used for future application in the diagnosis of a wide range of diseases which have gene marker in body fluids or tissue samples as an important alternative to the current used methods.

\section{Acknowledgements}

We gratefully acknowledge the EU, ERASMUS Life-Long Learning programme bilateral agreement for providing financial support for the study. 


\section{References}

[1] .Bertram, J. S., Molecular aspects of Medicine 21 (2000) 167.

[2]. Aminetzach, Y. T.; Macpherson, J. M.; Petrov, D. A., Science 309 (2005) 764.

[3]. Sawyer, S. A.; Parsch, J.; Zhang, Z.; Hartl, D. L., Proceedings of the National Academy of Sciences of the United States of America 104 (2007) 6504.

[4]. Nna, E.; Tothill, I.E.; Ludeman, L.; Bailey, T., Medical Principles and Practice 19 (2010), 433.

[5]. Olivier, M.; Eeles, R.; Hollstein, M.; Khan, M. A.; Harris, C. C.; Hainaut, P., Human Mutation 19 (2002) 607.

[6]. Behn, M.; Schuermann, M., Nucleic Acids Research 26 (1998) 1356.

[7]. Diamandis, E. P., Clinical Chemistry 46 (2000) 1523.

[8]. Montgomery, K. T.; lartchouck, O.; Li, L.; Loomis, S.; Obourn, V.; Kucherlapati, R., Current Protocols in Humman Genetics 7 (2008) 16.

[9]. Jianrong, C.; Yuqing, M.; Nongyue, H.; Xiaohua, W.; Sijiao, L., Biotechnology Advances 22 (2004) 505.

[10]. Drummond, T. G.; Hill, M. G.; Barton, J. K., Nature Biotechnology 21 (2003) 1192.

[11]. Jin, Y.; Yao, X.; Liu, Q.; Li, J., Biosensors and Bioelectronics 22 (2007) 1126.

[12]. Zhang, S.; Wright, G.; Yang, Y., Biosensors and Bioelectronics 15 (2000) 273.

[13]. Wei, F.; Qu, P.; Zhai, L.; Chen, C.; Wang, H.; Zhao, X. S., Langmuir 22 (2006) 6280 .

[14]. Tothill, I. E., Biosensors for cancer markers diagnosis, Seminars in Cell \& Development Biology 20 (2009) 55.

[15]. Gao, Z.; Zhang, J.; Ting, B. P., Biosensors and Bioelectronics 24 (2009) 1825.

[16]. Qureshi, A.; Kang, W. P.; Davidson, J. L.; Gurbuz, Y., Diamond and Related Materials 18 (2009) 1401.

[17]. Tombelli, S.; Minunni, M.; Luzi, E.; Mascini, M., Bioelectrochemistry 67 (2005) 135.

[18]. White, D. A.; Buell, A. K.; Dobson, C. M.; Welland, M. E.; Knowles, T. P. J., FEBS letters 583 (2009) 2587.

[19]. Sato, Y.; Sato, K.; Hosokawa, K.; Maeda, M., Analytical Biochemistry 355 (2006) 125. 
[20]. Zhong, X.; Leng, L.; Beitin, A.; Chen, R.; McDonald, C.; Hsiao, B.; Jenison, R. D.; Kang, I.; Park, S. H.; Lee, A., Nucleic Acids Research 33 (2005) 121.

[21]. Zhong, X.; Reynolds, R.; Kidd, J. R.; Kidd, K. K.; Jenison, R.; Marlar, R. A.; Ward, D. C., Proceedings of the National Academy of Sciences of the United States of America 100 (2003) 11559.

[22]. Ye, Y.; Wang, D.; Su, C.; Rong, T.; Guo, A., Genetics and Molecular Research 8 (2009) 1509.

[23]. Yakovchuk, P.; Protozanova, E.; Frank-Kamenetskii, M. D., Nucleic acids research 34 (2006) 564.

[24] Altintas, Z.; Uludag, Y.; Gurbuz, Y.; Tothill, I., Development of surface chemistry for SPR based Sensors for the Detection of Proteins and DNA molecules, Analytica chimica acta, 712 (2012) 138.

[25]. Hagihara, S.; Kumasawa, H.; Goto, Y.; Hayashi, G.; Kobori, A.; Saito, I.; Nakatani, K., Nucleic Acids Research 32 (2004) 278.

[26]. Jiang, T.; Minunni, M.; Wilson, P.; Zhang, J.; Turner, A. P. F., Biosensors and Bioelectronics 20 (2005) 1939.

[27]. Wang, R.; Minunni, M.; Tombelli, S.; Mascini, M., Biosensors and Bioelectronics 20 (2004) 598.

[28] Nakatani, K.; Sando, S.; Saito, I., Scanning of guanine-guanine mismatches in DNA by synthetic ligands using surface plasmon resonance, Nature biotechnology, 19 (2001) 51.

[29] Okumura, A.; Sato, Y.; Kyo, M.; Kawaguchi, H., Point mutation detection with the sandwich method employing hydrogel nanospheres by the surface plasmon resonance imaging technique, Analytical biochemistry, 339 (2005) 328.

[30] Dell'Atti, D.; Tombelli, S.; Minunni, M.; Mascini, M., Detection of clinically relevant point mutations by a novel piezoelectric biosensor, Biosensors and Bioelectronics, 21 (2006) 1876. 


\section{Biographies}

Zeynep Altintas $(\mathrm{PhD})$ received BSc degrees from "Molecular Biology and Genetics" and "Cell and Tissue Sciences" in 2007. She studied on neurodegenerative hereditary diseases in NDAL, Bogazici University between 2004 and 2008. She worked as full-time researcher and PhD student in Cranfield Health, Cranfield University, UK, from 2009-2010. She obtained her PhD degree at the Faculty of Natural Sciences and Engineering, Sabancı University, Istanbul, Turkey (February, 2012).

Ibtisam E.Tothill $(\mathrm{PhD})$ is an Associate Professor in Analytical Biochemistry and Head of the Advanced Diagnostics and Sensors Group at Cranfield Heath, Cranfield University, UK. Her research is focused on development of rapid sensors and diagnostics for health care, food and environmental analysis. 


\section{List of Figures}

Figure 1: Schematic representation of the applied experimental principle.

Figure 2: Sensorgrams of NeutrAvidin immobilization (a) and capture of singlestranded DNA sequences (scrambled sequence: grey line, wild-type target sequence: blue line, mutant-type target sequence: red line) using the Biacore 3000 system (b). Overall binding results of surface sequences to NeutrAvidin immobilized sensor surfaces (c). Bare-gold sensor chips were coated with 1\% MUDA to obtain a self assembled monolayer prior to the immobilization of NeutrAvidin. The biotinylated target and control (scrambled) sequences were captured by NeutrAvidin immobilised surface via the specific interaction between the NeutrAvidin and biotin.

Figure 3: Sensorgram of DNA hybridization using the Biacore 3000 instrument. The red line represents perfect match between mutant-type target surface and detection probe whilst the blue line shows one-point mismatch between wild-type target surface and detection probe. Gray line shows the non-specific binding on scrambled surface sequence (a). Overall results of DNA hybridization assay with confirmation of specificity and sensitivity (b). Linear regression and correlation coefficient of the results obtained in Biacore 3000 (c).

Figure 4: Comparison of DNA capture (a) and hybridization results obtained from the different probe surfaces to detect one-point mutation using the QCMA-1 biosensor (b). Linear regression and correlation coefficient of the results in QCMA-1 instrument (c). Bare-gold QCM chips were coated with 100\% MUDA to obtain a self assembled monolayer prior to the immobilization of NeutrAvidin on the surface. The biotinylated target and control (scrambled) sequences were captured by NeutrAvidin immobilised surface. The detection probe was designed according to the sequence of mutanttype target and it shows a perfect match with mutated sequence whereas it has a mismatch on wild-type target because of one nucleotide change.

Figure 5: Comparison of the two different sensor platforms for DNA capture (a) and hybridization assays (b). The used concentration of capture sequences (mutant-type target, wild-type target and scrambled control) were the same in both sensors (50 $\mu \mathrm{M})$. The hybridization efficiency of two sensors was compared for the reaction 
between mutant-type target surface and detection probe in the concentration range of $0.03-2 \mu \mathrm{M}$. 


\section{Figure 1}

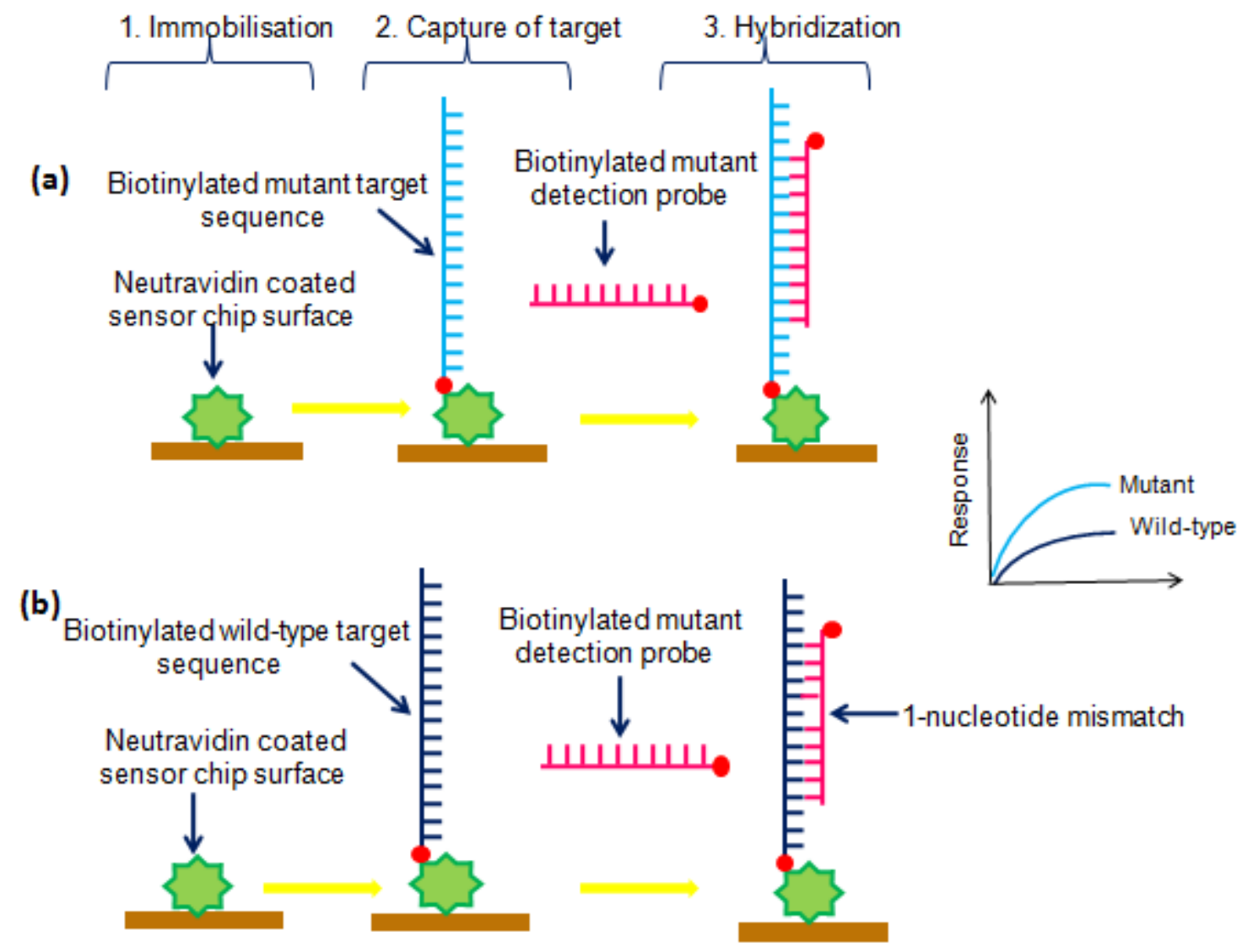


Figure 2

a)

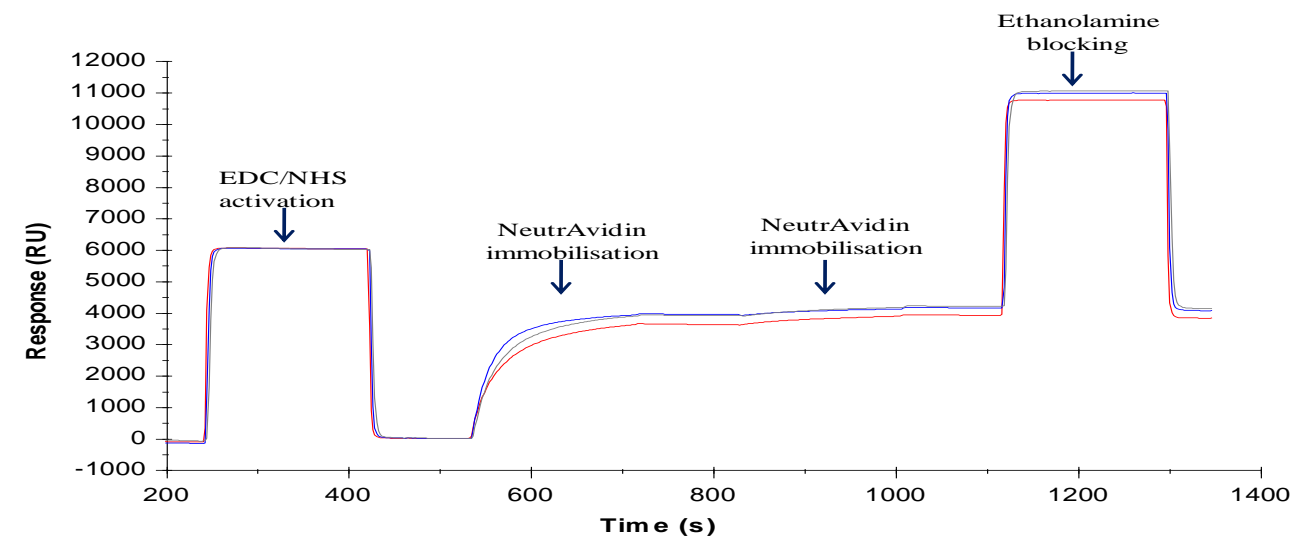

b)

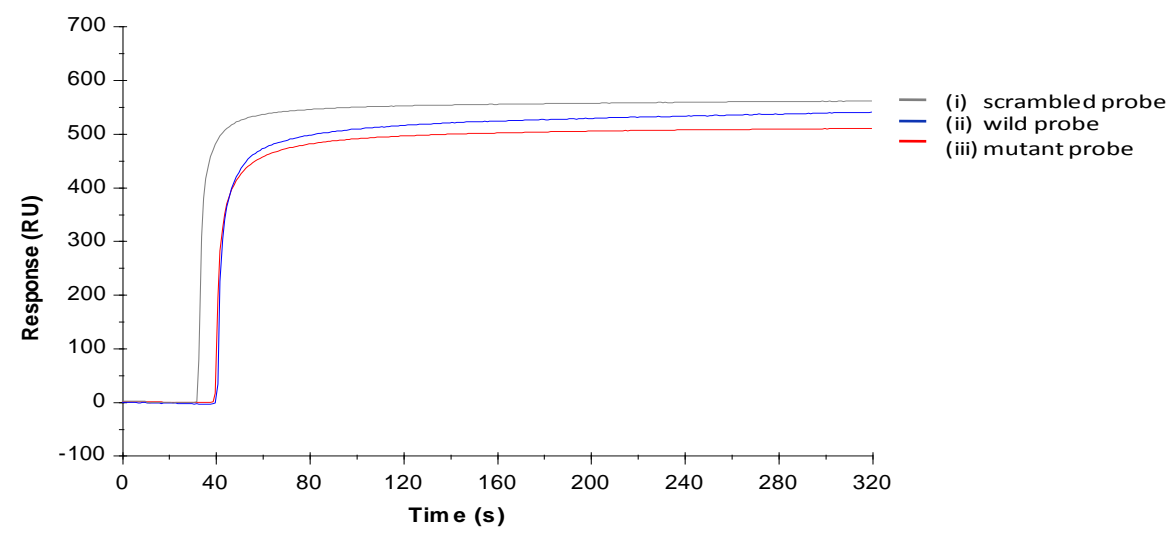

c)

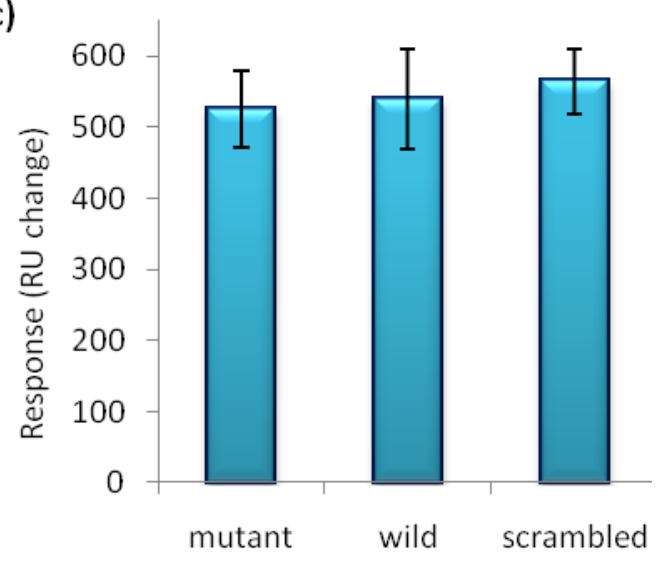

$\square$ Capture of DNA probes 
Figure 3

a)
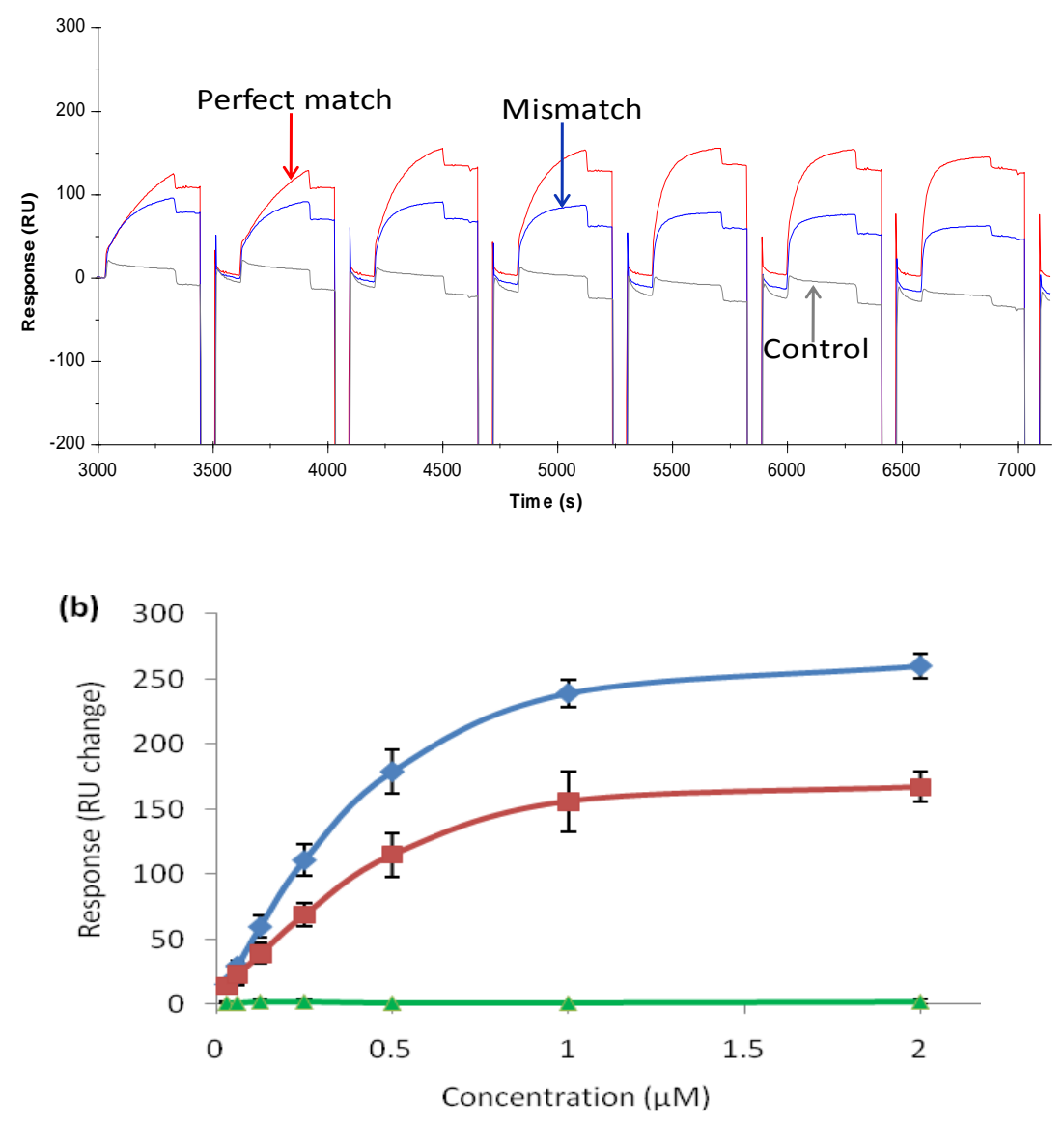

- Perfect match Mismatch due to SNP

- Non-specific hybridization

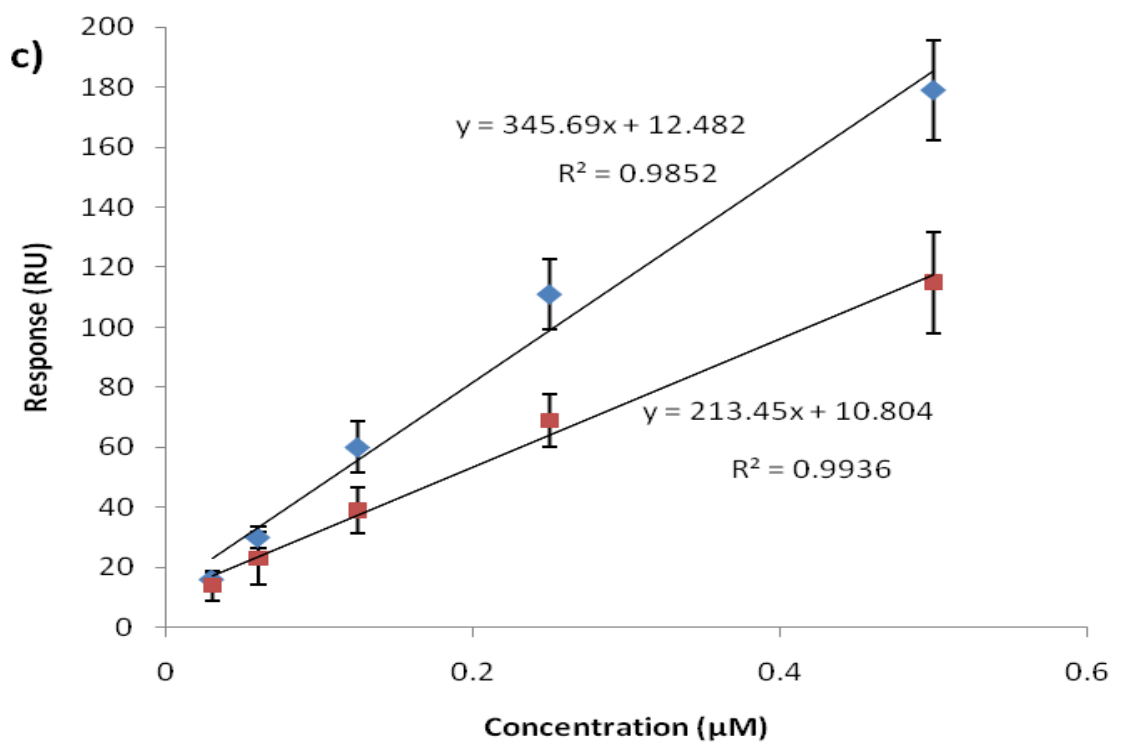

- Perfect DNA hybridization

- Mismatch due to SNP 
Figure 4:

a)

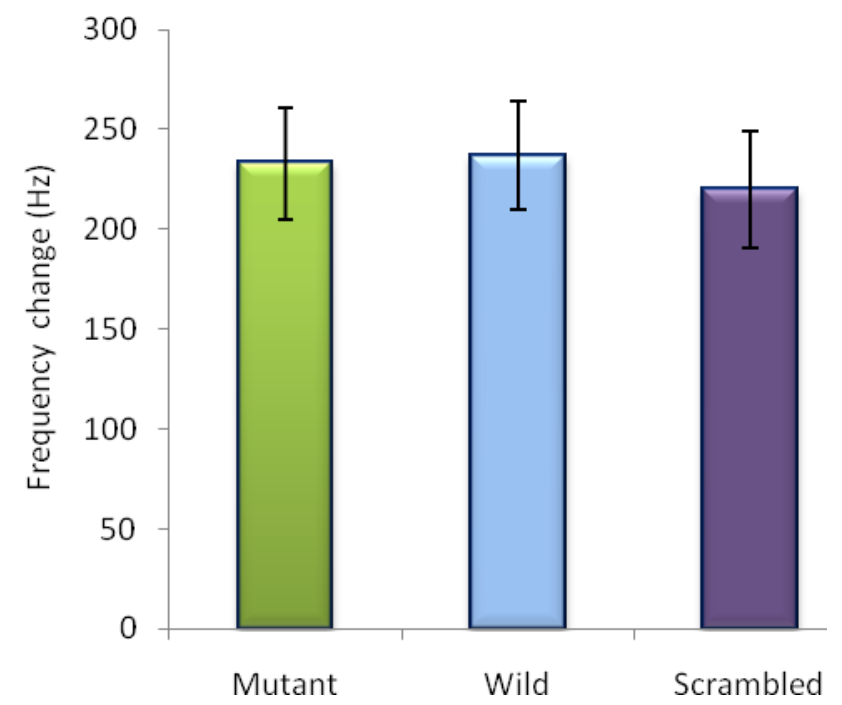

b)

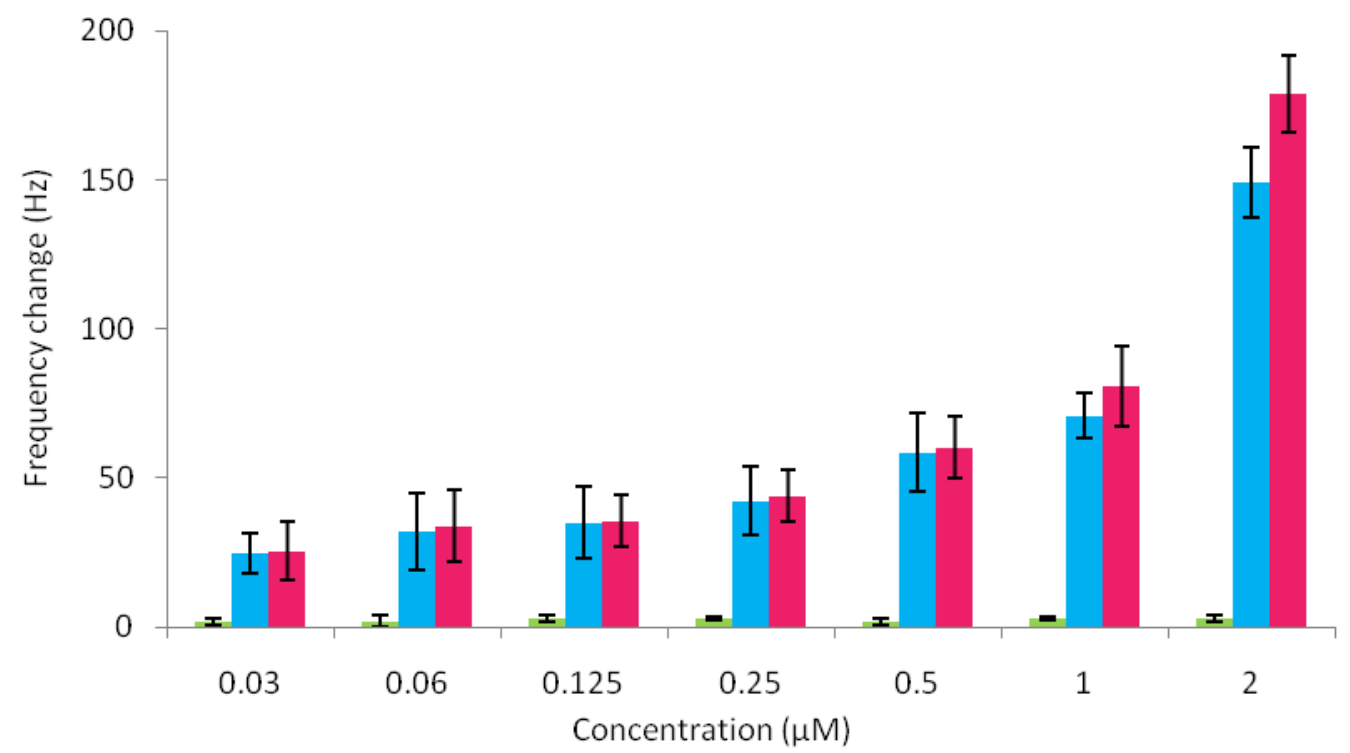

घybridization on scrambled probe surface $\quad$ One-point mutation on wild capture probe

- Perfect match on mutant capture probe 
c)

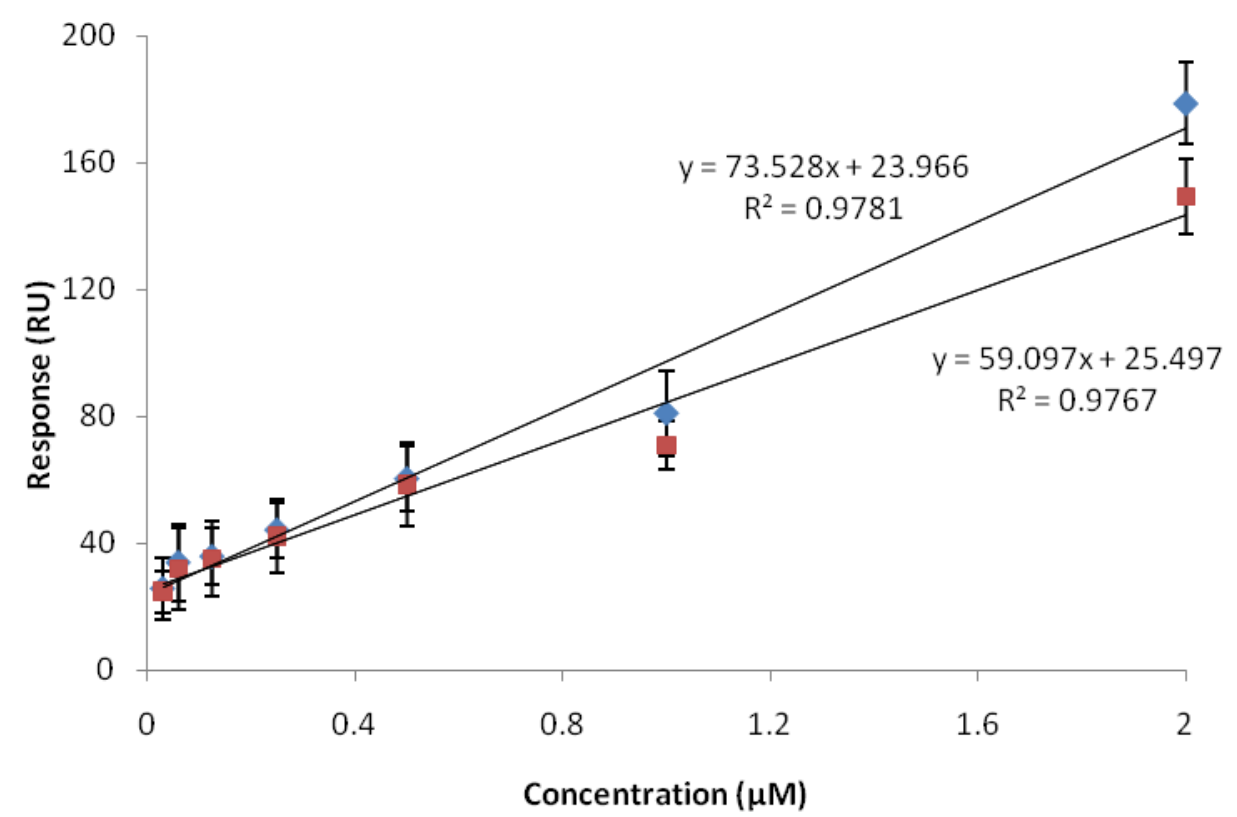

$\diamond$ Perfect DNA hybridization $\quad$ Mismatch due to SNP 
Figure 5:

a)

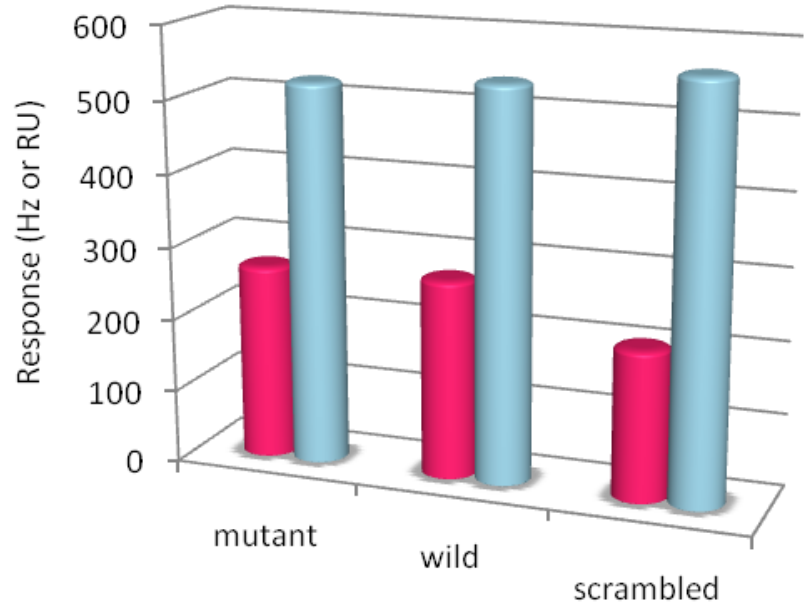

a Capture of probes on QCMA-1 sensor chip surface

$\square$ Capture of probes on Biacore 3000 chip surface

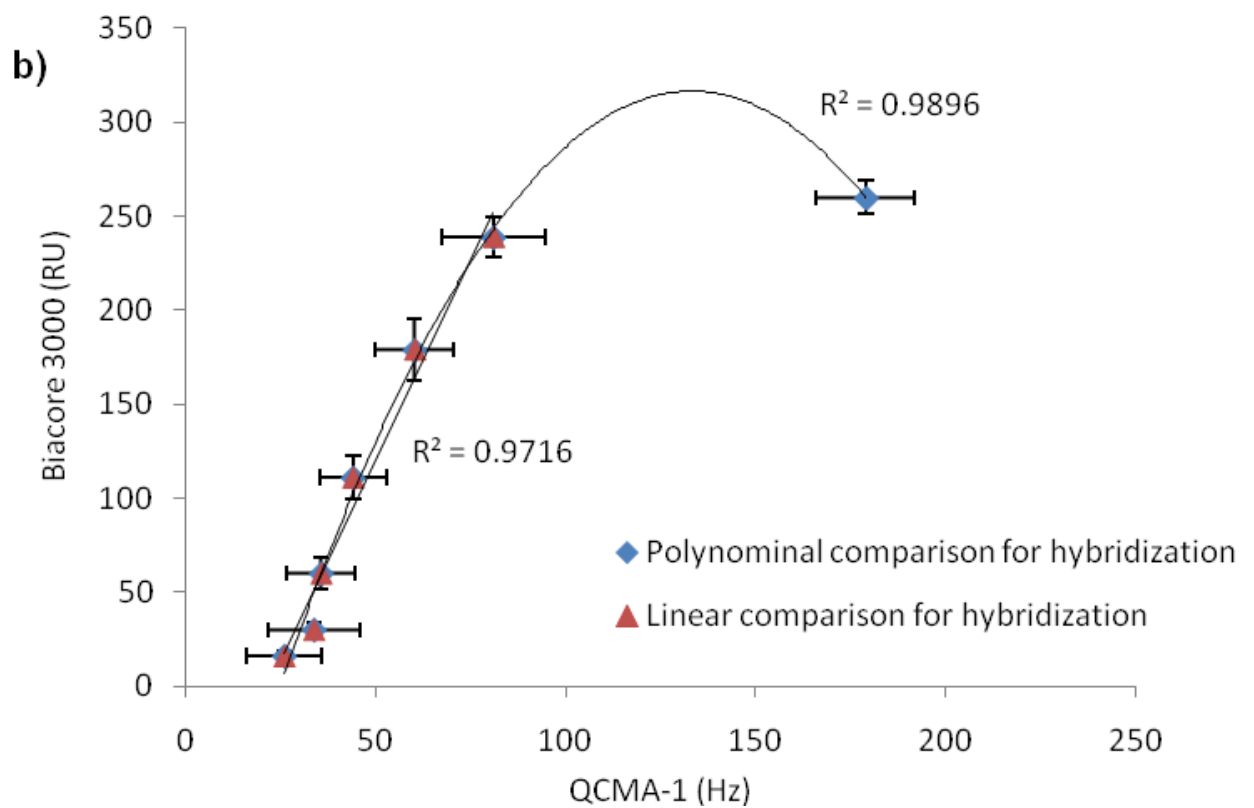

